\title{
Los Educadores y Educadoras Sociales en los Centros Educativos de Andalucía: Perfil y Desarrollo Profesional
}

\author{
Social Educators at Schools in Andalusia: Profile and \\ Professional Development
}

\author{
Eduardo S. Vila * \\ Pablo Cortés \\ Víctor M. Martín
}

Universidad de Málaga, España

\begin{abstract}
El artículo presenta parte de los resultados de un proyecto de investigación sobre el impacto de la implementación y la evolución del perfil profesional de los educadores y educadoras sociales en las escuelas de Andalucía. La metodología utilizada descansa en una perspectiva de corte evaluativo. En la misma, mediante una metodología mixta, se ha conjugado el análisis documental, los cuestionarios y los estudios de caso. A partir de ahí se desvelan los resultados vinculados el perfil, las funciones y el impacto que ha tenido mencionada figura en los centros educativos, teniendo en cuenta su desarrollo profesional en distintos niveles (legislativo, educativo y administrativo) y en relación a la valoración que hacen otros agentes educativos. Dichos resultados se articulan en torno a cuatro sub-apartados relacionados con las categorías definidas en este estudio: labor del educador-a, percepciones sobre y desde los educadores-as sociales, funciones y desempeño profesional y condicionantes de su labor y participación. Finalmente, se muestran algunas conclusiones en clave propositiva que muestran vías por las que seguir fortaleciendo esta necesaria figura en el sistema educativo, apostando por fortalecer las funciones que se perciben y manifiestan más necesarias en los centros e identificando las principales dificultades con las que se ha encontrado su labor.
\end{abstract}

Descriptores: Educación comunitaria; Escuela secundaria; Escuela primaria; Política educacional; Profesionales de la educación.

\begin{abstract}
This paper presents part of the results of a research project on the impact of the implementation and evolution of the professional profile of social educators at schools in Andalusia. The methodology used rests on an evaluative perspective. In this process, the documentary analysis, the questionnaires and the case studies have been combined by a mixed methodology. In this point, the results linked to the profile, functions and impact of the social educator are revealed, taking into account their professional development at different levels (legislative, educational and administrative) and in relation to the assessment that make other educational agents. These results are articulated around four sub-sections related to the categories defined in this study: the work of the educator, perceptions about and from the social educators, functions and professional performance and conditioning factors of their work and participation. Finally, some conclusions are shown in a proactive key that show ways to continue strengthening this necessary figure in the education system, betting on strengthening the functions that are perceived and manifested most necessary in the centers and identifying the main difficulties with which found his work.
\end{abstract}

Keywords: Community education; Secondary schools; Primary schools; Educational policy; Educational population.

*Contacto: eduardo@uma.es

ISSN: $1696-4713$

www.rinace.net/reice/

revistas.uam.es/reice
Recibido: $\quad 2$ de abril 2019

$1^{\text {a }}$ Evaluación: 20 de mayo 2019

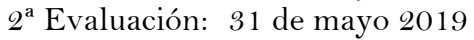

Aceptado: $\quad 8$ de junio 2019 


\section{Introducción}

Los numerosos cambios sociales y políticos que se están produciendo en los últimos años en materia de inclusión social, educación, inmigración, políticas de dependencia, etc. (Cortés, Rivas y Leite, 2016), están provocando, a su vez, la necesidad de que los centros educativos se transformen y se reinventen con el objetivo de atender a las nuevas demandas y fenómenos sociales, incluyendo la manera en que se producen las relaciones educativas (Vila, 2019). Para dar respuesta a estos cambios, entre otras cuestiones, se ha producido la incorporación de nuevos programas y profesionales a los centros escolares, entre los que se encuentran en Andalucía los educadores y las educadoras sociales, siendo el objeto de nuestro estudio.

En este escenario, la escuela adquiere unos compromisos como institución clave a la hora de atender y abordar acciones contra los procesos de exclusión social y educativa desde diversas dimensiones: axiológicas, instrumentales, procedimentales, etc., que requiere su conexión y colaboración con otros agentes socio-educativos como son las familias, servicios sociales, centros de tutela u otras administraciones públicas. Todo ello ha hecho pertinente la incorporación de estos profesionales al sistema educativo, como piezas fundamentales de concienciación ciudadana y como nexo entre el mundo escolar y el comunitario (Cendrero et al., 2012; Rico y Martín, 2014).

\section{Revisión de la literatura}

En Andalucía (BOJA nº 213 de 3 de noviembre de 2006) se realizó en 2006 una convocatoria para incorporar a educadoras y educadores sociales al sistema educativo. Profesionales cualificados para trabajar en contextos educativos y socioculturales diversos, cuyo perfil profesional les capacita para conjugar e integrar la dimensión escolar del alumnado con otras dimensiones del sujeto, tales como la personal, la familiar y la comunitaria. Esto supone una aportación significativa, ya que abre nuevos escenarios de intervención escolar tradicionalmente demandados desde una mirada educativa y en pro del desarrollo integral del alumnado (Apple y Beane, 1997; Dewey y Dewey, 1962). Su incorporación al mismo tuvo lugar en el curso 2007-08, persiguiendo desde la Administración que las educadoras y educadores sociales se convirtieran en motores de cambio y transformación de dicho sistema, como consecuencia de acercar lo escolar al territorio y a la comunidad (Rico, 2012).

En los últimos 20 años hemos vivido una situación de acercamiento y progresiva cooperación entre la institución escolar y sus pedagogías, y la pedagogía social (Romans, Petrus y Trilla, 2000). En este contexto, se viene tendiendo a convertir los centros escolares en territorios de acción, también, de educadores y educadoras sociales, replanteando con ello ciertos esquemas propios de la cultura escolar y, siempre de manera complementaria, de la propia función docente y educativa.

$\mathrm{Al}$ poner el foco aquí, y tal y como ya se adelantó en Sierra y otros (2017), descubrimos que son escasos los trabajos de investigación en el panorama español que vienen preguntándose sobre la incidencia de la figura del educador y educadora social en los centros educativos. Situación debida en parte a la relativa juventud de esta figura (Sáez, 2003; Sáez y Molina, 2006), y en parte a la heterogeneidad de perfiles profesionales que, en cada contexto autonómico, vienen desempeñando unas funciones similares. Desde la 
revisión aludida y ampliando la misma, podemos agrupar las aportaciones identificadas en tres grupos:

- Un corpus de textos de naturaleza teórica, en los que se discute acerca del sentido epistemológico y pedagógico dentro de la 'tradicional' distinción entre pedagogía escolar y pedagogía social, y cómo ese debate es llevado al contexto práctico de la incorporación de educadores y educadoras sociales a los centros educativos (Jiménez, 2013; Ortega, 2005; Parcerisa y Forés, 2003; Pericacho, 2014; Terrón, Cárdenas y Rodríguez, 2017).

- Textos de experiencias, en los que se narran las prácticas de educadores y educadoras sociales, exponiendo las formas de intervención y reflexionando sobre su alcance educativo, sus debilidades y fortalezas, así como investigaciones incipientes y precursoras en este ámbito (González, Olmos y Serrate, 2015, 2016; Ruiz, 2013; Serván, 2013; Terrón et al., 2015).

- Experiencias de formación en la Universidad (Hernández y Tolino, 2013). Dentro de este grupo predominan los trabajos centrados en el seguimiento de las prácticas y sus posibilidades formativas (Beloki et al., 2011; Morales, 2013).

Desde una perspectiva histórica, Parcerisa (2008) nos recuerda que Extremadura y Castilla-La Mancha (en 2002) fueron las Comunidades Autónomas pioneras en la implantación de educadoras y educadores sociales a los centros escolares. Comunidades a las que se sumó Andalucía en 2006, donde en la actualidad se cuenta con un total de 66 educadores/as, un número claramente poco significativo para el volumen de centros educativos en la Comunidad Autónoma Andaluza y las necesidades sociales que acarrean. En otras Comunidades encontramos otras iniciativas o experiencias, vinculadas sobre todo a proyectos de colaboración entre administraciones locales y educativas, y entidades del tercer sector, como son el caso de Aragón, Galicia, Cataluña, País Vasco, Baleares, Valencia o Castilla y León, con diferentes matices e importancia (Castillo, Galán y Pellissa, 2012)

Además, centrándonos en el caso andaluz, de las resoluciones específicas para legislar la contratación de dichos profesionales, la Ley de Educación de Andalucía (2007) contempló en su Título III la intervención de otros profesionales (diferentes a los docentes) para optimizar la atención educativa al alumnado con necesidades específicas de apoyo educativo. Ese mismo año, a través del Decreto 19/2007, de 23 de enero, se estableció que los Equipos de Orientación Educativa adscribirán a sus puestos de trabajo al personal funcionario con la titulación de educador social.

Por desgracia, el tiempo transcurrido desde entonces no ha servido para que se lleve a cabo el proceso de generalización de esta figura profesional en los centros educativos andaluces que se anunció en su momento como declaración de intenciones. En todo caso, ante la escasez de investigaciones al respecto en Andalucía, nos hemos detenido en estudiar el impacto educativo y social de la implantación del educador social en los centros educativos, entrando a conocer y evaluar la naturaleza y alcance de las intervenciones desarrolladas y la evolución de este novedoso perfil profesional en los centros educativos. Bajo ese propósito, esta investigación se ha focalizado, en primer lugar, en el análisis documental (normativa y literatura científica), lo que nos permitió desarrollar un estado de la cuestión (Sierra et al., 2017); y, en segundo lugar, en la evaluación de la 
implementación de los mencionados profesionales. En esta línea, los objetivos de este estudio son los siguientes:

- Estudiar el impacto de la presencia de estos profesionales en los centros educativos de Andalucía respecto de sus tres principales focos de actuación: la comunidad, la mejora de la convivencia y la lucha frente al absentismo escolar.

- Analizar el perfil, funciones y desarrollo profesional del educador social en los centros educativos.

- Conocer estas realidades para poder realizar propuestas de mejora a nivel profesional e institucional.

- Promover el reconocimiento profesional, social e institucional de estos profesionales.

A partir de estos objetivos, hemos encontrado cuatro bloques temáticos sobre los que pivotan los resultados del estudio, de los cuales en el presente artículo nos centramos en explicar los relacionados con los perfiles y el desarrollo profesional de los educadores y educadoras sociales en las escuelas de Andalucía, encontrando hallazgos interesantes que nos hacen repensar cómo se está aplicando esta necesaria, aunque poco explotada, orden legislativa.

Para ello, coincidimos con la consideración de entender las profesiones, entre ellas las educativas, como construcciones sociales (Caride, 2002), cuya puesta en práctica está intrínsecamente relacionada con sus funciones que, a su vez, se encuentra vinculada al contexto de desarrollo de su labor profesional (Sáez, 2003) y las personas o colectivos hacia las cuales se dirige la misma. En este sentido, aunque existen multitud de referencias sobre competencias y funciones de los educadores-as sociales (Bautista-Cerro y Melendro, 2011; Herrera, 2010; Martín y Quiroz, 2009; Molina y Blázquez, 2006; Pérez, 2009; Riera, 1998; Románs, Petrus y Trilla, 2000; Vallés, 2009, 2011), resulta necesario profundizar en las mismas de forma contextualizada en los centros educativos debido al contexto tan particular en el que se encuentran. En este sentido, nos parecen acertadas las palabras de Caride (2002) cuando afirma que para ser y actuar competentemente se requiere un saber, un saber hacer y un saber estar. Por eso, a la luz de la bibliografía existente (Alguacil, 2012; Barranco, Díaz y Fernández, 2012; Castillo, 2012; Castillo y Galán, 2008; Castro y Valín, 2007; Gimeno, 2009; Jiménez, 2013; González, Olmos y Serrate, 2015, 2016; Laorden, Prado y Royo, 2006; López Zaguirre, 2014; Longás, 2000; Ortega, 2008 y 2014; Terrón, Cárdenas y Rodríguez, 2017) y los resultados obtenidos en este estudio, consideramos importante tratar de superar la separación entre educación formal, no formal e informal, abogando por eliminar las barreras para el trabajo coordinado e interdisciplinar.

\section{Método}

Este proyecto de investigación descansa en una perspectiva de corte evaluativo (Kushner, 2000; Shaw, 2003), que mediante una metodología mixta (Caride y Fraguela, 2015) conjuga análisis documental, cuestionarios y estudios de caso (Simmons, 2011). Esto se hace con el fin de evaluar, por un lado, el alcance socio-educativo de las políticas públicas en torno a la presencia de las y los educadores sociales en los centros educativos de 
Andalucía y, por otro lado, el desarrollo de su perfil e identidad profesional. El diseño de la investigación consta de dos fases para la recogida de datos.

Para la primera fase, intensiva, se referencia el análisis de la bibliografía y legislación existente al respecto y la elaboración de un cuestionario, a través de una metodología no experimental y descriptiva (Kerlinger y Lee, 2002). Ambos recursos nos permiten conocer las actividades y funciones que realizan los educadores sociales en los centros escolares, así como un balance de la materialización en la práctica escolar.

Sobre el cuestionario cabe matizar que se trata de una encuesta estructurada (autoadministrada online), con preguntas cerradas, de alternativas múltiples, escalas tipo Likert e ítems de respuesta abierta. Se invitó a participar a los 66 educadores y educadoras sociales que había a nivel andaluz en 2016 y participó aproximadamente el $40 \%$ de ellos. Con esto intentamos paliar el efecto de los errores de muestreo, mediante la aplicación a toda la población. Además, también tratamos de reducir los errores de medida estandarizando las condiciones de aplicación y con instrucciones que garantizaran la igualdad de condiciones para todas y todos los encuestados. En cuanto a validez de contenido, se garantiza a través de la valoración por jueces expertos sobre los enunciados de las preguntas planteadas. Para ello, el primer borrador fue valorado, a partir de criterios de claridad y pertinencia, por un equipo formado por expertos académicos y profesionales de la educación social que enriquecieron con sus aportaciones dicha encuesta. El cuestionario final se estructura en cuatro bloques: 1) información básica-perfil, 2) contexto laboral y coordinación, 3) desempeño profesional, 4) proyecciones de futuro y propuestas de mejora, y 5) comentarios finales.

Contextualizando la muestra, debemos, asimismo, indicar que los resultados obtenidos corresponden a 26 educadores y educadoras sociales con edades comprendidas entre los 33 y los 59 años, de los cuales 14 son hombres y 12 son mujeres. En cuanto al número de respuestas de educadores según provincias, tenemos que en Córdoba respondieron dos, en Málaga seis, en Cádiz cinco, en Almería uno, en Sevilla siete, en Granada dos, en Huelva uno y en Jaén dos.

De todos los encuestados, dos trabajan en departamentos de orientación (en un IES cada uno) y el resto (24) se ocupan de atender los centros escolares de Primaria adscritos del Equipo de Orientación Educativa de zona que les corresponde (los centros a los que atienden varían entre dos y ocho). En referencia a la formación inicial de los encuestados, todos afirman estar titulados/diplomados en Educación Social, salvo una, que está habilitada como educadora. Cinco de ellos están colegiados. En cuanto a la antigüedad del puesto en el que están, la mayoría afirma llevar cinco años (12), otros contestaron llevar seis años (3), siete años (7), ocho años (1) y el resto afirma llevar tres años o menos (3). Y respecto a la experiencia previa de los mismos, estos son los más destacados: Protección de menores (4); Animación sociocultural (3); Drogodependencias (2); Tercera edad (2); Servicios sociales comunitarios (2). Otros ámbitos que también han sido señalados son: Menores drogodependientes, Reforma juvenil, Cooperación internacional, Educación de calle, Absentismo escolar y Atención a personas con diversidad cognitiva. Estos perfiles tan variados son significativos y, en todo caso, se encuentran relacionados con las amplias funciones definidas para ellos.

La segunda fase, extensiva, consiste en el desarrollo de dos estudios de caso en profundidad en dos centros educativos de distintas localidades andaluzas y seleccionados por criterios de idoneidad, diversidad y representatividad en función de los objetivos de la 
investigación. Para el desarrollo de cada estudio caso, primero se han realizado entrevistas en profundidad a los siguientes informantes: educador/a social, orientador/a, miembro del equipo directivo, docente, alumnos/as y padres/madres. Segundo, se han realizado grupos focales con agentes de las distintas profesiones educativas. Y, tercero, se ha hecho una recopilación de documentos e información en cada centro en torno a indicadores de convivencia y académicos para su análisis de los últimos 8 años.

Para el proceso analítico de esta segunda parte, esto es el Análisis de Datos Cualitativos, hemos recurrido al análisis interpretativo como estrategia metodológica y a la Teoría Fundamentada (Trinidad, Carrero y Soriano, 2006), a la hora de profundizar en las categorías interpretativas. Para ello, se desarrolla un sistema de selección de temas y categorías emergentes con el apoyo del programa informático Atlas-ti. En el proceso de tematización se obtienen 2404 ítems de interés relacionados con el foco, los cuales confluyen en siete unidades de análisis o categorías, que a continuación se exponen:

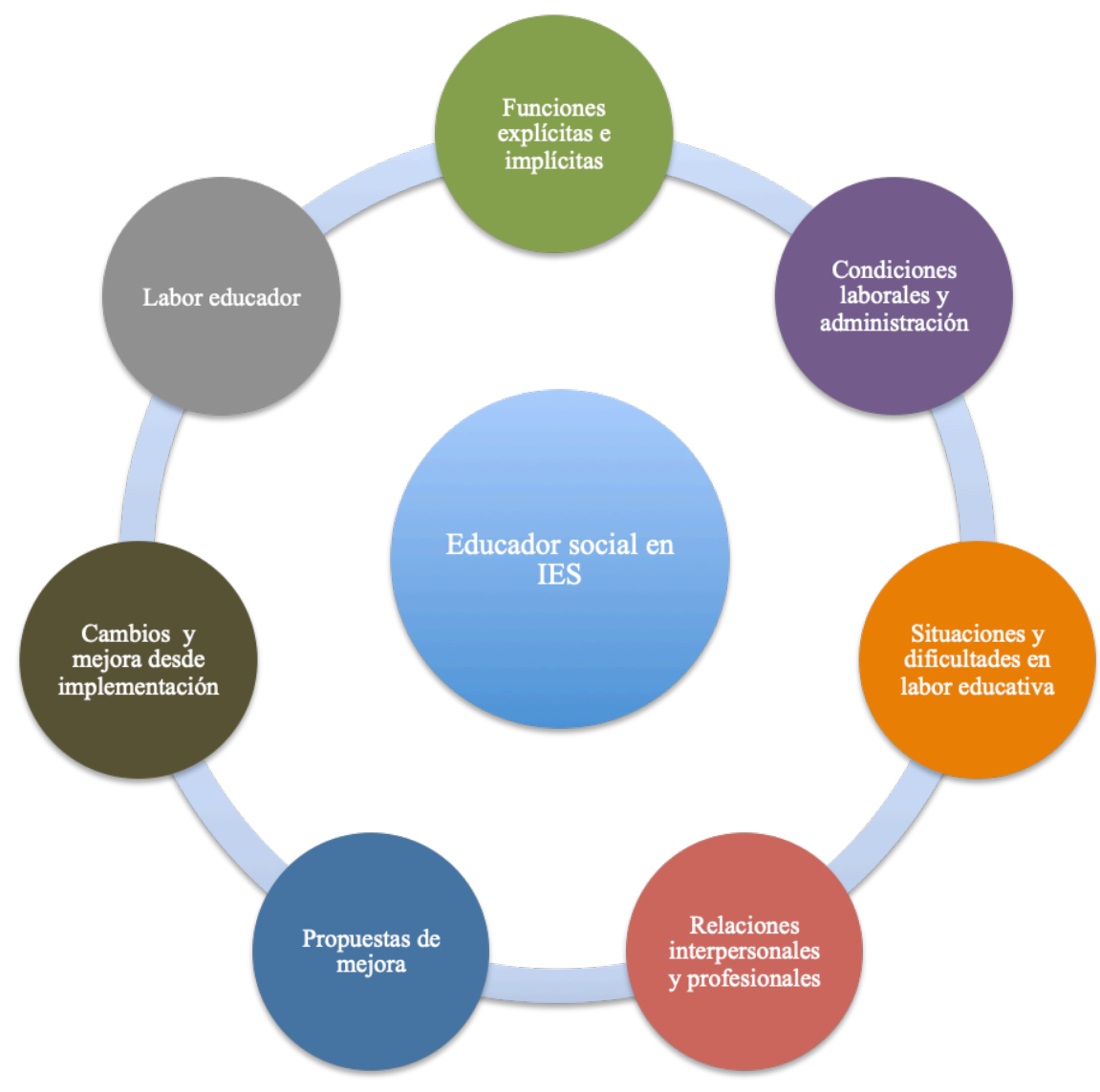

Figura 1. Categorías de análisis

Fuente. Elaboración propia.

Así, a partir del análisis y categorización de la información obtenida por los distintos instrumentos antes descritos, destacamos, a continuación, los principales hallazgos y evidencias vinculadas al objetivo sobre el perfil, funciones y desarrollo profesional en los centros educativos de los educadores y educadoras sociales, por lo que nos centramos en el análisis triangulado de resultados desde los ítems del cuestionario relacionados con el mismo y las palabras expresadas por los informantes en los estudios de caso. También 
cabe decir que se establecen una serie de sub-apartados para clarificar la presentación de dichos resultados, relacionados con las categorías definidas.

\title{
3. Resultados
}

\subsection{Labor del educador-a}

Una de las primeras ideas relacionadas con la presencia y funciones de las educadoras y educadores sociales en los centros educativos tiene que ver con la necesidad de ir más allá de lo académico en los centros educativos. Este es uno de los aspectos más sobresalientes corroborados desde los resultados de las encuestas y los estudios de caso, donde el profesorado y otros agentes educativos coinciden en ello. De forma ilustrativa se indica en una de las entrevistas de los estudios de casos: "El profesor llega hasta los deberes. El educador más allá”. Y en otra entrevista, se explicita lo siguiente:

\begin{abstract}
A ver cómo me explico yo... To he tenido aquí, y tengo, alumnos que sé que académicamente no van a conseguir nada, y alumnas, pero a lo mejor si ese alumnado tiene relaciones sexuales y es capaz de acordarse de que tiene que poner medios, de que tiene que ser consciente de que quiere, de que no tiene que hacerlo por fuerza y tal... he trabajado más ese tipo de cosas a que obtengan el título. Sí, con los que no van a obtener el título trabajo eso y con otros trabajos otra clase de parámetros de su vida personal que le va a servir para su vida personal, porque académicamente no quieren, no es que no puedan, no quieren. (Educador IES)
\end{abstract}

\subsection{Percepciones sobre y desde los educadores-as}

Otros aspectos de interés basados en las evidencias encontradas tienen que ver con el grado de conocimiento y aceptación de su labor y presencia en los centros, así como sus propias percepciones en torno a sus funciones en los mismos. En referencia a esto, hemos encontrado disparidad en la visión. Por un lado, en las entrevistas en un centro de compensatoria, una estudiante expresa,

La labor de X, es, como, por ejemplo, ayudar a menores, adultos... darles consejos, intentar ayudarles como está haciendo X conmigo, intentar que sus problemas vayan disminuyendo. (Estudiante IES)

Por su lado, una directora aporta lo siguiente:

\begin{abstract}
$X$ es fundamental, simplemente yo detecto que está poco tiempo (...) Debería extenderse muchos más días. Es verdad que hay centros, que no sean de compensatoria, que a lo mejor no son tan necesarios, pero en los centros de compensatoria absolutamente son necesarios todos los días, todas las jornadas. De hecho, nos apoya muchísimo porque aqui estamos... Tengo una orientadora, tengo un jefe de estudios y está $x$ (educador) y cada uno tiene funciones y no están parados. (...). En principio, para mi sería el nexo que nos falta entre el centro y la familia. Hay veces que nosotros no podemos llegar a las familias, ahí están las asistentes sociales pero ese nexo... que aqui hemos tenido un problema, hemos hablado con el niño... pero luego queremos saber qué pasa con la familia, que actuación ha habido... Es verdad que están los Servicios Sociales, pero no están dentro de mi centro, nos dan información, pero es información, sin embargo, mi educador social, si es mi educador social. (Directora IES)
\end{abstract}

Como podemos ver, la figura del educador es vista como esencial. Esto contrasta con la visión del propio educador o educadora, a los cuales en algunos de los apartados del cuestionario se les preguntó por aspectos o cuestiones relacionadas con el trabajo realizado en los centros escolares y la valoración y percepción que tienen del mismo. Asimismo, pudimos observar cómo cuando les preguntamos sobre si sus compañeros 
saben qué es lo que hace un educador social en el centro escolar, un $48 \%$ de los participantes señala un nivel medio (3) en su respuesta y un $36 \%$ un nivel bajo (2). Por tanto, parece que hay algo de información, pero no suficiente. Y, en todo caso, lo que sí se constata es que ha habido una evolución y que los comienzos fueron difíciles, porque no se sabían muy bien sus funciones e incluso hubo quien los consideraba una especie de "espías de la administración”, según palabras de una directora. De ese desconocimiento y desconfianza inicial se ha evolucionado a una valoración muy positiva por parte de la totalidad de la comunidad educativa de su labor, si bien sigue habiendo sobre todo en el sector docente personas que no terminan de comprender su papel y el que no estén en las clases. Como manifestaba un educador: "Nos hemos tenido que ir ganando el sitio".

Siguiendo con este tema, se puede observar como sólo el 12,5\% afirma sentir con totalidad que cuentan con él o ella para el desarrollo de la mayor parte de las actividades programadas en el proyecto de centro; un 50\% siente lo mismo, aunque en menor grado y el resto, $37,5 \%$ muestra en su respuesta una valoración baja o muy baja acerca de este tema. Todas las respuestas en torno a esta cuestión van en la misma línea y reflejan como más de la mitad de los participantes señala con alto grado de valoración (medio y alto) el hecho de participar en proyectos y actividades programas en sus centros escolares. Al respecto, cabe mencionar cómo encontramos que muchas de las veces el educador social no está el tiempo suficiente en los centros, lo que le imposibilita que se pueda involucrar en el equipo educativo. Es más, aún no están considerados en la plataforma de la Junta de Andalucía como parte del grupo docente/educador del centro. Apunta una directora entrevistada:

La pena es que no podamos contar con más frecuencia con él (...) realmente yo sí veo la utilidad y su labor, pero cuando debe atender a otros centros y no dispone de suficientes horas, es difícil. (Directora IES)

\subsection{Funciones y desempeño profesional}

Este aspecto también se ve reforzado en las respuestas que dieron acerca del nivel de satisfacción que tienen en relación a su desempeño profesional y a las tareas que acometen: un $44 \%$ parece sentir alto grado de valoración y un $36 \%$ un nivel medio de satisfacción, frente a un $12 \%$ que está poco satisfecho y un $8 \%$ nada satisfecho. Esta satisfacción profesional manifiesta, refrendada en las entrevistas, es un elemento de interés pues implica también el interés profesional en vencer las dificultades encontradas como perfil nuevo en los centros educativos y que subraya un aspecto constitutivo de su identidad profesional respecto a cómo se ha ido construyendo.

La otra cuestión anunciada, vinculada a sus funciones, nos invita a indicar, en primer lugar, que sí es cierto que las funciones que les atribuye la legislación vigente son muy amplias, si bien teniendo presente la Resolución original de 16 de octubre de 2006, de la Dirección General de Gestión de Recursos Humanos, por la que se realiza convocatoria para la cobertura provisional durante el curso 2006-2007 en puestos de educación social en el ámbito educativo, las principales son:

- Intervención en la aplicación de medidas dirigidas al alumnado para la mejora de la convivencia y colaboración con el profesorado en la consecución de tal fin.

- Seguimiento del alumnado absentista.

- Mediación en conflictos: alumnado-centro-familia. 
- Desarrollo de programas para la educación de valores y la integración multicultural.

En esta línea, una de las cuestiones importantes que se ha tratado dentro del cuestionario ha sido la percepción que tienen los educadores sociales acerca de sus funciones dentro de la propia institución escolar. En este caso, la respuesta ha sido variada: referente al nivel de valoración que tienen acerca del cumplimiento de sus funciones profesionales, el 50\% de los participantes ha señalado cumplirlas en un nivel medio, mientras que el 33,3\% afirma cumplir con las funciones totalmente. Solo un $4,2 \%$ señala cumplir con sus funciones con un nivel de valoración bajo y un 12,5\% no cumplir con las funciones que han de realizar dentro de los centros escolares a los que están destinados.

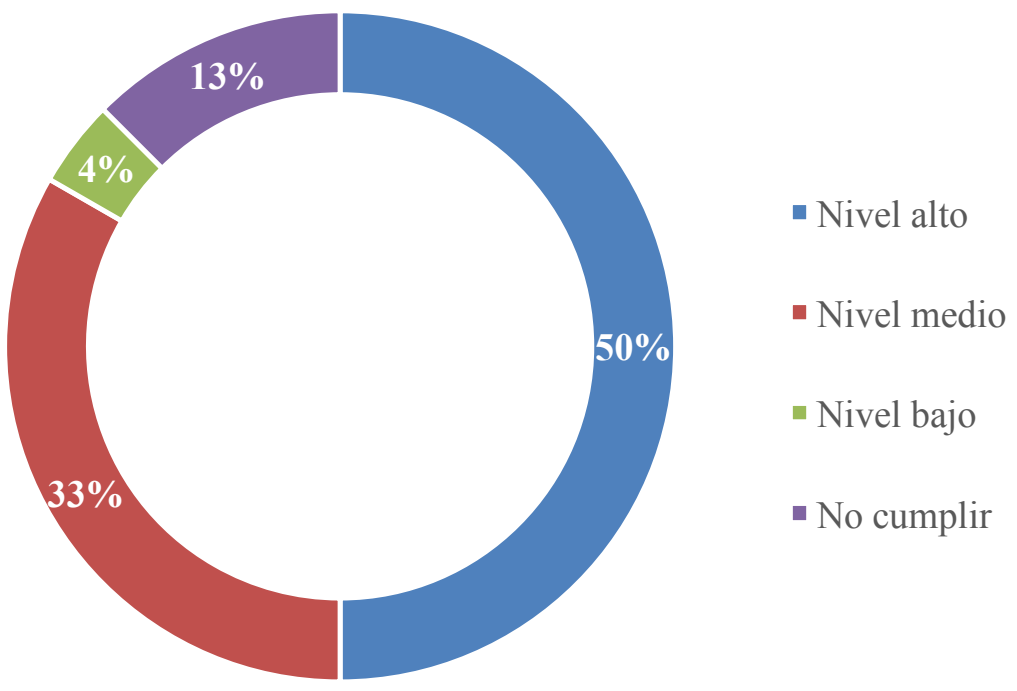

Figura 2. Nivel de valoración cumplimiento de funciones

Fuente. Elaboración propia.

Como se observa de manera mucho más clara en la figura 2 , se observa cómo la mitad de los y las educadoras sociales afirman no realizar o poder cumplir plenamente sus funciones, lo cual es un dato significativo.

Por otro lado, aunque algo más de la mitad de los encuestados (54\%), sostiene desde un nivel medio de valoración el hecho de considerar que abordan correctamente sus funciones, el porcentaje aumenta en detrimento de esta cuestión, valorando con un nivel bajo, un 16\%, y muy bajo, un $25 \%$, el hecho de que sean abordadas adecuadamente las funciones que han de realizar en los centros. En este mismo sentido, sus valoraciones con respecto a la pregunta de si consideran que el centro escolar da cobertura a sus funciones profesionales, sólo un $8,3 \%$ señala que no se da cobertura, mientras que un $41 \%$ y un $37 \%$ tiene una valoración medio-baja acerca de esta cuestión; no obstante, un 12,5\% si que considera con un nivel alto de valoración que el centro escolar en el que están da cobertura a sus funciones profesionales.

Asimismo, como se aprecia en la legislación y documentación analizada y se constata en esta investigación, convivencia, participación e interculturalidad aparecen como ámbitos fundamentales para el desarrollo del perfil profesional de los educadores y educadoras sociales en los centros educativos andaluces. En esta línea, en base a lo estipulado en las Instrucciones de 2010 de la Dirección General de Participación e Innovación Educativa 
de la Junta de Andalucía, por las que se regula la intervención del educador y educadora social en el ámbito educativo, se indica en el siguiente cuadro cuáles de esas funciones son realmente las que se dan en los centros educativos en mayor medida, desde las evidencias mostradas a partir del análisis de los cuestionarios y los estudios de caso.

Cuadro 1. Funciones desarrolladas en los centros educativos

\begin{tabular}{|c|c|}
\hline FUNCIONES LEGALMENTE DEFINIDAS & $\begin{array}{l}\text { MÁS DESARROLLADAS EN } \\
\text { LOS CENTROS }\end{array}$ \\
\hline $\begin{array}{l}\text { Realizar el seguimiento del alumnado absentista con } \\
\text { intermediación entre la familia y el centro }\end{array}$ & $\mathrm{x}$ \\
\hline $\begin{array}{l}\text { Mediar en conflictos: alumnado-centro, familia-centro y } \\
\text { alumnado-familia }\end{array}$ & $\mathrm{x}$ \\
\hline $\begin{array}{l}\text { Intervenir con el alumnado y las familias en problemas de } \\
\text { convivencia }\end{array}$ & $\mathrm{x}$ \\
\hline Trabajar como mentor del alumnado en situaciones de riesgo & $\mathrm{x}$ \\
\hline $\begin{array}{l}\text { Organizar y desarrollar actividades de formación de las familias } \\
\text { del alumnado en situación de riesgo }\end{array}$ & \\
\hline $\begin{array}{l}\text { Coordinar actividades extraescolares para el alumnado en } \\
\text { situación de riesgo }\end{array}$ & \\
\hline Realizar el seguimiento de actividades no lectivas del alumnado & \\
\hline Colaborar en programas de vida saludable & \\
\hline Organizar y desarrollar programas socioeducativos & \\
\hline $\begin{array}{l}\text { Desarrollar actividades de ocio y tiempo libre con el alumnado en } \\
\text { situación de riesgo }\end{array}$ & \\
\hline $\begin{array}{l}\text { Desarrollar programas de habilidades sociales, comunicativas y } \\
\text { para la relación con el alumnado en situación de riesgo }\end{array}$ & $\mathrm{x}$ \\
\hline Desarrollar programas para la integración multicultural & $\mathrm{x}$ \\
\hline Desarrollar programas de educación en valores & $\mathrm{x}$ \\
\hline $\begin{array}{l}\text { Coordinar los recursos de la zona para el uso por parte del } \\
\text { alumnado en situación de riesgo }\end{array}$ & $\mathrm{x}$ \\
\hline $\begin{array}{l}\text { Colaborar con el profesorado en el funcionamiento del aula de } \\
\text { convivencia }\end{array}$ & $\mathrm{x}$ \\
\hline $\begin{array}{l}\text { Realizar cuantas otras actuaciones y actividades que a } \\
\text { requerimiento de la Administración Educativa sean necesarias }\end{array}$ & \\
\hline
\end{tabular}

La realidad es que se trata de muchas y variadas funciones, de las cuales en unos centros se hace más énfasis en unas u otras en relación a las necesidades, las prioridades y los contextos. Lo que sí está claro es que el perfil profesional sí podríamos decir que parte de estos ejes: (1) intervenciones directas con el alumnado y/o sus contextos; y (2) el desarrollo de programas específicos dirigido a sectores concretos de alumnado. Además, lo que nos trae también la presencia de estos profesionales en los centros educativos son las posibilidades de trabajar colectivamente y de manera coordinada con los contextos de referencia, por lo que entendemos esta figura como fundamental para apoyar la buena marcha de los centros, cubrir necesidades que los demás profesionales de la educación no pueden hacer y vehicular propuestas de índole social y comunitaria que no se ven fortalecidas precisamente porque las dinámicas de funcionamiento de los centros educativos suelen estar más encorsetadas y con una perspectiva más centrada en lo académico. Como plantea un orientador en una entrevista: "Tienen una información fresca 
y real de cada niño. El profesorado no podemos llegar donde llega el educador: servicios sociales, familias, protección de menores".

Esta situación a medio camino entre agente comunitario y personal del equipo educativo genera no pocas dificultades; pero no sólo los otros profesionales sino también las familias y, sobre todo, el alumnado, sabe reconocer su labor y funciones. Desde las familias se considera que: "en los centros de compensatoria son necesarios todos los días." Y desde el alumnado, frases tan significativas como éstas: "Me ayuda a que controle mis nervios" o "Intenta comprenderte. Entender los problemas".

En todo caso, de todas las funciones manifiestas y latentes la realidad hace que se centren sobre todo en las tareas relacionadas con la convivencia y el absentismo, a veces con más posibilidades reales y otras como apaga-fuegos, expresión que se ha reiterado en diversas entrevistas.

\subsection{Condicionantes de su labor y participación}

Otra cuestión que es necesario destacar son las cuestiones percibidas como condicionantes de las tareas que desarrollan y su desarrollo profesional. En este sentido, supone motivo de queja del colectivo la burocratización y "protocolarización" (aplicación de protocolos a menudo poco flexibles) de su labor en gran parte, y la poca valoración que sienten por parte de la Administración Educativa. Si a esto unimos el carácter itinerante de sus puestos en la mayoría de los casos, siendo lo común que estén en al menos dos centros educativos, observamos tres de los mayores condicionantes constatados de su labor, lo cual dificulta además sobremanera las posibilidades de coordinación en los centros educativos. Respecto a esto último, indica un orientador: "Si viene sólo dos días, no puede involucrarse en un proyecto. Cuantos más recursos, mejor. Por eso es una buena figura”.

Asimismo, también se indaga sobre si consideran o no necesario ampliar sus funciones profesionales. En este caso no hay una respuesta única más o menos destacada: un $20 \%$ indica no estar nada de acuerdo, un 33,3\% señala estar entre poco de acuerdo en un nivel de valoración bajo, otro 33,3\% en un nivel medio y solo un 12,5\% afirma estar totalmente de acuerdo. En este mismo sentido, parece haber más o menos consonancia con las respuestas que ofrecen cuando les preguntamos si consideran que se deberían cambiar las funciones a nivel legislativo. En este caso, un $45 \%$ afirma con un nivel medio y un $25 \%$ con un nivel alto de valoración, la necesidad de cambiarlas; frente a un $25 \%$ con un nivel bajo y un $4,2 \%$ que señala que no es necesario cambiar las funciones desde lo legislativo.

Desde esta óptica, y recuperando la información de las entrevistas, parece que no es tanto una cuestión de reformas legislativas respecto a sus funciones, aunque algunas las consideran ambiguas, sino de posibilidades para abarcarlas o cumplirlas en un contex to a menudo de itinerancia y con poca vinculación orgánica con los centros educativos. Esta es, probablemente, la principal reivindicación del colectivo y los profesionales que conviven con ellos y ellas, lo cual incluiría aspectos como unas mejores condiciones laborales que pasan también por su inclusión en los equipos de orientación y los consejos escolares para mejorar sus posibilidades de influencia en los mismos. Esto es debido a que normalmente su participación en las reuniones de los centros (desde claustros hasta sesiones de evaluación) es 'voluntaria' y sus aportaciones por contra se consideran muy importantes por parte de los equipos docentes y directivos por las aportaciones que realizan en las mismas. 
Sobre esta cuestión las familias y el alumnado tienen una apreciación coincidente, pues en todos los casos se valora el impacto positivo a lo largo del tiempo de la presencia de estos profesionales, sobre todo en lo referente al absentismo y abandono escolar y la convivencia en los centros. Este hecho se corrobora con los datos documentales ofrecidos por los centros colaboradores en esta investigación (en todos los casos las tasas de absentismo y abandono, así como otros indicadores como los partes disciplinarios, han disminuido considerablemente) y los de otros proporcionados por otros educadores y educadoras sociales.

\section{Discusión y conclusiones}

En este apartado final, cabe comenzar diciendo, completando lo indicado anteriormente, que las políticas autonómicas andaluzas dieron un primer paso en la apuesta por la inclusión de esta figura educativa en los centros de enseñanza, pero se detecta una falta de mantenimiento y apuesta por estos profesionales en el tiempo, puesto que no se han vuelto a dar más convocatorias ni se ha incrementado el número de educadores-as. En todo caso, y desde la perspectiva del objetivo en el que centramos los resultados de este artículo, hay que destacar determinados elementos de interés surgidos desde el mismo y los resultados expuestos anteriormente.

Terrón, Cárdenas y Rodríguez (2017) manifiestan en su estudio que la mayoría de los educadores-as sociales suelen estar en los centros educativos de secundaria. Sin embargo, en nuestra investigación la mayoría afirma encontrarse adscritos a equipos de orientación educativa de zona y atender de forma itinerante un número variables de centros, que incluían tanto de secundaria como de primaria, con un criterio más vinculado a territorios de influencia y características de los mismos que al nivel educativo del centro. Consideramos muy importante que se fomente esa presencia también en la educación primaria para que sus funciones se puedan desarrollar desde los primeros cursos de la escolaridad obligatoria, con las posibilidades a nivel preventivo, mediador, comunitario y de gestión de la convivencia que esto conlleva.

De esta manera, hay que destacar que se percibe con claridad que cuando se conoce y reconoce su labor, los educadores-as cumplen un papel fundamental en los centros educativos (no solo - desde una mirada pedagógica- en los centros de compensatoria), puesto que hace de puente entre las situaciones, nuevos fenómenos y casuísticas sociales (que se materializan de manera directa e indirecta en los centros educativos), y las necesidades formativas y educativas del alumnado y el propio profesorado y equipo directivo. Esto supone dar cumplimiento a lo que Castillo y Galán (2008, p. 65) denominan el "paso de una óptica curricular a una perspectiva socioeducativa en la escuela." En esta línea, se ve una clara apuesta por enfatizar la necesidad de fortalecer una óptica más comunitaria e inclusiva frente al carácter instructivo del sistema educativo. Así, el desfase histórico al que se ve sometida la escuela reclama un reajuste de sus funciones, destacando la incorporación de la figura de las y los educadores sociales como profesionales necesarios y definiendo ámbitos y funciones que tienen como trasfondo la aspiración por hacer un sistema más equitativo. Esta afirmación se aprecia con rotundidad en la evolución de la discusión teórica sobre el foco y el encargo de la pedagogía/educación social, en especial cuando sucede en el marco de la institución escolar, así como se desprende de los resultados de los cuestionarios y diversas alusiones en los estudios de caso. 
Por lo tanto, se trata de una especie de profesión bisagra. Por eso, como ha apuntado Ortega (2014), la incorporación de los educadores sociales a los centros escolares no puede ser solo un recurso profesional más, sino la posibilidad de relación necesaria entre éstos y la comunidad. En relación a esto, algunos autores definen la profesión bajo la imagen de un "caballero andante" (Vallés, 2009) o de un "hombre orquesta" (Herrera, 2010), expresiones que con variantes han emergido en el transcurso de la investigación, hablando de la labor de estos profesionales de forma positiva y enfatizando ese carácter complejo, aunque necesario de la misma.

Igualmente, resulta de interés respecto a las funciones analizadas la disonancia que se produce a veces entre lo normativo y la realidad. Ya observamos en el cuadro 1, desarrollado en el anterior apartado, qué funciones de las definidas legalmente son las que se desarrollan profesionalmente en el día a día de los centros, al hilo de los resultados encontrados. En todo caso, no deja de ser también interesante fijarnos en las diferencias entre la percepción ajena (otros profesionales, familias, ...) y la propia (de los educadoresas sociales), destacando por parte de estos últimos la pugna que afirman haber desarrollado en los centros educativos para obtener reconocimiento profesional, sobre todo por parte del resto de compañeras y compañeros, desde una situación de desconocimiento e incomprensión inicial a una valoración muy positiva sobre el impacto de su presencia y ejercicio profesional en los centros, siendo casi unánime la petición de una mayor presencia en los mismos.

Otro aspecto a destacar, ya emergente en Sierra y otros (2017), es la relación entre las concepciones sobre lo educativo y la cultura profesional, donde la dialéctica entre la labor pedagógica y de atención a la diversidad y la labor instructiva se percibe insistentemente en la bibliografía y se constata en los resultados. De hecho, una aportación que parece fundamental es que tanto los educadores-as sociales como el resto de profesionales entienden que la presencia de los primeros supone un eslabón que une estas visiones, que permite el diálogo entre las mismas y que sugiere estrategias para la cooperación en los centros educativos desde sus funciones, tanto explícitas como implícitas. En este sentido, Sáez (2005) indica cómo el desarrollo profesional depende en la figura del educador-a de aspectos como el conocimiento que se es capaz de construir (sobre todo el práctico y actitudinal); la autonomía y responsabilidad en la acción; el reforzamiento y/o modificación de la imagen pública; y el papel que tiene las personas implicadas en el proceso educativo. En este sentido, este colectivo posee un conocimiento profesional de gran valía reconocida en los centros educativos y del que se carece formativamente en otras profesiones educativas. Además, sus funciones hacen que tengan autonomía sin dejar de lado la interdependencia inherente a la mayoría de los procesos educativos fuera del ámbito instructivo y sin que esto suponga un menoscabo de su responsabilidad profesional (un valor que en general defienden incluso con vehemencia, como se reflejó en los cuestionarios y grupos focales, sobre todo). Esto hace que su imagen haya mejorado también de forma sustancial en los centros, como comentamos anteriormente, así como que ese reconocimiento haga que sean demandados en otros centros, según la mayoría de los informantes ha afirmado.

En el apartado de reivindicaciones vinculadas a sus condiciones laborales y de desempeño profesional, la necesidad de adscripción a un centro educativo y eliminación de la itinerancia como referente mayoritario en el colectivo, es un punto importante para mejorar su impacto e imagen en los centros, además de facilitar el trabajo colaborativo con la comunidad, las familias, el profesorado y demás profesionales relacionados dentro 
y fuera de las escuelas e institutos. A esto podemos sumar el hecho de no contar con un perfil específico y con atribuciones apropiadas en el programa de gestión para profesionales de la educación de la Junta de Andalucía (Séneca) y el carácter voluntarista de su participación en reuniones colegiadas en los centros (solicitan mayoritariamente que pudieran estar adscritos a los centros como otros profesionales), lo que suponen limitaciones para un mejor desarrollo de sus funciones.

En definitiva, a raíz de los resultados obtenidos y las conclusiones anteriores, resulta importante tener presente estos planeamientos:

- El educador-a social poco a poco deja de verse en estos contextos sólo como recurso para emergencias o conflictos, sino que se está integrando, a pesar de las dificultades y limitaciones, en una dinámica de trabajo cooperativo para la mejora de los centros y la comunidad educativa.

- Se trata de un agente educativo clave también por la información que puede proporcionar a los centros educativos de los contextos, los colectivos y las personas. Por ello es el profesional más adecuado para trazar puentes entre la cultura escolar y las formas culturales presentes en los mismos.

- Puede proporcionar al profesorado estrategias y recursos para trabajar con alumnado diverso y en situación de riesgo social para contribuir a la mejora de la calidad educativa, de la convivencia y del derecho a la educación.

Al margen de esto, quisiéramos destacar que esta investigación cuenta con limitaciones inherentes a la muestra de los cuestionarios, la cual, siendo significativa, parte de un total de sujetos no muy numeroso. También sería de interés ampliar el número de estudios de casos e incluir a responsables de la Administración Educativa como informantes para tener más referentes.

Además de lo anterior, es necesario incidir en que esta investigación nos hacer ver la necesidad de desarrollar más estudios que profundicen en las prácticas profesionales de los educadores-as en los centros educativos. Esto con el propósito, no de buscar las fallas en la correspondencia entre las funciones delimitadas normativamente y el ejercicio profesional, sino, en el sentido personal (entendido como singular y puesto en contexto) que el ejercicio profesional comporte: Es decir, vislumbrar la complejidad de las relaciones que se entablan, las fricciones que se viven con la cultura institucional y docente en los centros educativos, las particularidades de las relaciones pedagógicas que merece la pena entablar y sostener; cuestión ésta donde la presencia de estos profesionales ofrece referentes más dialógicos, desde una perspectiva freireana.

Por último, insistir en que la realidad es que se trata de muchas y variadas funciones las de los educadores y educadoras sociales, de las que, en unos centros se hace más énfasis en unas u otras en relación a las necesidades, las prioridades de cada contexto. En relación a todo esto, lo que nos trae también la presencia de estos profesionales en los centros educativos es, por un lado, no olvidar que la calidad de la educación está íntimamente relacionada con la calidad de los espacios de relaciones (González y San Fabián, 2018), y con la convivencia; y, por otro lado, las posibilidades de trabajar colectivamente y de manera coordinada con los contextos de referencia. De ahí que entendamos esta figura como fundamental para apoyar la buena marcha de los centros, cubrir necesidades que los demás profesionales de la educación no pueden hacer y vehicular propuestas de índole social y comunitaria que no se ven fortalecidas precisamente porque las dinámicas de 
funcionamiento de los centros educativos suelen descansar en una perspectiva centrada en lo académico.

\section{Referencias}

Apple, M. y Beane, J.A. (1997). Escuelas democráticas. Madrid: Morata.

Alguacil, E. (2012). El educador social en el sistema educativo: Competencias profesionales para la intervención sistémica. Sinergias, 1, 29-37.

Barranco, R., Díaz, M. y Fernández, E. (2012). El educador social en la educación secundaria. Valencia: Nau Libres.

Bautista-Cerro, M. J. y Melendro, M. (2011). Competencias para la intervención socioeducativa con jóvenes en dificultad social. Educación XX1, 14(1), 179-200.

https://doi.org/10.5944/educxx 1.14.1.268

Beloki, N., Ordeñana, B., Darreche, L., González, M. N., Flecha, A., Hernando, M. C., ... y Sanz, Z. (2011). Innovando el practicum de educación social: Una experiencia de trabajo colaborativo. Revista de Educación, 354, 237-264.

Caride, J. A. (2002). La pedagogía social en España. En V. Núñez (Ed.), La educación en tiempos de incertidumbre: Las apuestas de la pedagogía social (pp. 88-112). Barcelona: Gedisa.

Caride, J. A. y Fraguela, R. (2015). Cuando el proyecto se hace método: Nuevas perspectivas para la investigación socioeducativa en red. Pedagogía Social. Revista Interuniversitaria, 26, 139172. https://doi.org/10.7179/PSRI_2015.26.06

Castillo, M. (2012). La intervención del educador social en el marco educativo formal. Barcelona: UOC.

Castillo, M. y Galán, D, (2008). El papel de los educadores sociales en los centros de secundaria: Una propuesta para el debate. Educación Social. Revista de Intervención Socioeducativa, 38, 121133.

Castillo, M., Galán, D. y Pellissa, B. (2012) La incorporació dels educadors socials a l'escola: L'experiència present $i$ les perspectives de futur. Barcelona: UOC.

Cendrero, J., Jiménez, R., Rodríguez, M. L. y Rubio, F. (2012). El educador social como profesional del sistema educativo y su desarrollo en Andalucía. Revista Orión, 6, 37-48.

Cortés, P., Rivas, J. I. y Leite, A. (2016). Education and social change in Spain: From crisis to opportunity. Ethnography and Education, 11 (2), 204-22 1.

https://doi.org/10.1080/17457823.2016.1147971

Dewey, J. y Dewey, E. (1962). Schools of tomorrow. Nueva York, NY: E.P. Dutton.

Gimeno, S. (2009). La figura del educador social en centros de educación primara en el ámbito de la animación sociocultural. Quaderns D'Animació i Educació Social, 10, 11-12.

González, M., Olmos, S. y Serrate, S. (2015). Pensamiento y acción socioeducativa en contextos de enseñanza secundaria. Un estudio descriptivo-correlacional. Teoría de la Educación. Revista Interuniversitaria, 27(2), 91-114. https://doi.org/10.14201/teoredu201527291114

González, M., Olmos, S. y Serrate, S. (2016). Análisis de la práctica profesional del educador social en centros educativos de secundaria. Pedagogía Social. Revista Interuniversitaria, 28, 229-243. https://doi.org/10.7179/PSRI_2016.28.17

González, M. T. y San Fabián, J. L. (2018). Buenas prácticas en medidas y programas para jóvenes desenganchados de lo escolar. REICE. Revista Iberoamericana sobre Calidad, Eficacia y Cambio Educativo, 16(2), 41-60. https://doi.org/10.15366/reice2018.16.2.003 
Hernández, M. A. y Tolino, A. C. (2013). Promoción de la figura del educador social en la escuela desde el practicum de educación social en la Universidad de Murcia. RES, Revista de Educación Social, 16, 1-17.

Herrera, D. (2010). Un acercamiento a la situación formativa de las educadoras y educadores sociales. Revisa de Educación, 353, 641-666.

Jiménez, R. (2013). Los educadores y educadoras sociales en el sistema educativo formal. Una mirada sobre los orígenes, funciones y propuestas de futuro. Sinergias, 1, 81-106.

Kerlinger, F. y Lee, H. (2002). Investigación del comportamiento. Métodos de investigación en ciencias sociales. Ciudad de México: McGraw-Hill.

Kushner, S. (2000). Personalizar la evaluación. Madrid: Morata. https://doi.org/10.4135/9781849209557

Laorden, C., Prado, C. y Royo, P. (2006). Hacia una educación inclusiva. El papel del educador social en los centros educativos. Pulso, 29, 77-93.

Longás, J. (2000). Educación social y escuela. Educación Social. Revista de Intervención Socioeducativa, 15, 101-106.

López Zaguirre, R. (2014). Las educadoras y los educadores sociales en los centros escolares en el estado español. Revista de Educación Social, 16, 1-6.

Martín, M. T. y Quiroz, C. (2009). Perfil y funciones del educador social. En E. López-Barajas (Coord.), Estrategias de formación en el siglo XXI (pp. 285-316) Barcelona: Ariel.

Molina, J. y Blázquez, R. (2006). El educador social en la educación secundaria. Revista de Educación Social, 32, 39-58.

Morales, S. (Coord.) (2013). El practicum como experiencia de aprendizaje en educación social. Propuestas para su desarrollo: Panificación, tutoría docente y proyección personal. Madrid: Universitas.

Ortega, J. (2005). La educación a lo largo de la vida: La educación social, la educación escolar, la educación continua... Todas son educaciones formales. Revista de Educación, 338, 167-175

Ortega, J. (2014). Educación social y enseñanza: Los educadores sociales en los centros educativos, funciones y modelos. Edetania. Estudios y Propuestas Socio-Educativas, 45, 11-31.

Parcerisa, A. (2008). Educación social en y con la institución escolar. Pedagogía Social, Revista Interuniversitaria, 15, 15-27. https://doi.org/10.7179/PSRI_2008.15.02

Parcerisa, A. y Forés, A. (2003). Didáctica y educación social: ¿Una convivencia llena de posibilidades? Revista de Educación Social, 25, 71-84.

Pérez, G. (2009). Pedagogía social-educación social. Construcción científica e intervención práctica. Madrid: Narcea.

Pericacho, J. (2014). Educación social y modelos escolares alternativos: Aproximación teórica, retos y posibilidades socioeducativas. Revista de Educación Social, 18, 1-15.

Rico, J. I. (2012). Educación social en el ámbito educativo, una mirada desde los colegios profesionales. Sinergias, 1, 15-28.

Rico, J. I. y Martín, V. M. (2014) La educación social en territorios periféricos. Valencia: Nau Llibres.

Riera, J. (1998). Concepto, formación y profesionalización del educador social, el trabajador social y el pedagogo social. Valencia: Nau Llibres.

Romans, M., Petrus, A. y Trilla, J. (2000). De profesión: Educador/a social. Barcelona: Paidós.

Ruiz, M. (2013). El educador social en la escuela. Un ejemplo de intervención desde un enfoque intercultural. Revista de Educación Social, 16, 1-14. 
Sáez, J. (2003). La profesionalización de los educadores sociales: En busca de la competencia educativa cualificadora. Madrid: Dykinson.

Sáez, J. (2005). La socialización de los profesionales. La construcción de la identidad profesional del educador social. En C. Mínguez (Coord.), Educación social: Discurso, práctica y profesión (pp. 99-146). Madrid: Dykinson.

Sáez, J. y Molina, J. G. (2006). Pedagogía social. Pensar la educación social como profesión. Madrid: Alianza.

Serván, C. (2013). La fábrica y la tribu. Conflictos y prácticas de convivencia en un centro de secundaria. Sinergias, 2, 149-157.

Shaw, I. (2003). La evaluación cualitativa. Barcelona: Paidós.

Sierra, J. E., Vila, E. S., Caparrós, E. y Martín, V. M. (2017). Rol y funciones de los educadores y las educadoras sociales en los centros educativos andaluces. Revista Complutense de Educación, 28(2), 479-495. https://doi.org/10.5209/rev_RCED.2017.v28.n2.49542

Simmons, H. (2011). El estudio de caso: Teoría y práctica. Madrid: Morata.

Terrón, T., Rebolleda, T., Rodríguez, M., Esteban, M. (2015). La diversidad cultural desde la perspectiva de los/as educadores/as sociales: Un estudio en los centros educativos de Andalucía. Educatio Siglo XXI, 33(2), 141-164. https://doi.org/10.6018/j/233181

Terrón, T., Cárdenas R. y Rodríguez, R. (2017). Educación intercultural inclusiva. Funciones de los/as educadores/as sociales en instituciones educativas. Pedagogía social. Revista Interuniversitaria, 29, 25-40. https://doi.org/10.7179/PSRI_2017.29.02

Trinidad, A., Carrero, V. y Soriano, R. (2006). Teoría fundamentada. Grounded theory, la construcción de la teoría a través del análisis interpretacional. Madrid: CIS.

Vallés, J. (2009). Manual del educador social. Intervención en servicios sociales. Madrid: Pirámide.

Vallés, J. (2011). Análisis y valoración de las funciones de los educadores sociales en España. Tesis doctoral. UNED.

Vila, E. S. (2019). Repensar la relación educativa desde la pedagogía de la alteridad. Teoría de la Educación. Revista Interuniversitaria, 31(2), en prensa. https://doi.org/10.14201/teri.20271

\section{Breve CV de los autores}

\section{Eduardo S. Vila}

Profesor Titular en Teoría e Historia de la Educación de la Universidad de Málaga. Ha sido también Maestro de Educación Primaria y Coordinador Provincial de Educación Intercultural en Málaga. Miembro del Grupo de Investigación HUM-169 "Teoría de la Educación y Pedagogía Social”. Sus principales líneas de investigación son educación intercultural, pedagogía social, políticas educativas, cultura de paz y educación para los derechos humanos, teoría de la educación e igualdad de género. Posee más de 150 publicaciones, entre artículos científicos (de los cuales 15 están en revistas JCR/ESCI y/o SCOPUS), libros y capítulos de libro. Ha sido ponente en numerosos Congresos y Seminarios, ha participado en 15 proyectos de investigación y 12 proyectos de innovación educativa, obteniendo en 2009 el Premio a la Innovación Educativa de la UMA (mención especial). También ha realizado estancias de investigación y participado como evaluador de proyectos en varios países latinoamericanos. Actualmente, es Coordinador del Master 
Oficial en Cambio Social y Profesiones Educativas. ORCID ID: https://orcid.org/ooooo002-8598-7654. Email: eduardo@uma.es

\section{Pablo Cortés}

Profesor Contratado Doctor en el Departamento de Didáctica y Organización escolar de la Universidad de Málaga. Miembro del grupo de investigación consolidado ProCIE y de la BSA (British Sociological Association). Sus líneas de investigación versan en torno a las narrativas, la identidad profesional de los agentes educativos, las prácticas disruptivas y las ecologías del aprendizaje, teniendo más de medio centenar de artículos y capítulos de libro sobre dichas temáticas. Asimismo, participa activamente en tres redes de investigación en educación a nivel nacional e internacional: Reunid y Retinde (España) y REDICA (México). ORCID ID: https://orcid.org/0000-0002-9604-044X. Email: pcortes@uma.es

\section{Víctor M. Martín}

Profesor Contratado Doctor en el Departamento de Teoría e Historia de la Educación y MIDE de la Universidad de Málaga, donde desarrolla su tarea docente e investigadora, que gira en torno a la Pedagogía Social, Educación Social, procesos educativos en el ámbito penitenciario, educación intercultural, exclusión social, cultura de paz y derechos humanos. Es Doctor en Pedagogía por la Universidad de Málaga y ha sido educador social en Instituciones Penitenciarias. Cuenta con más de un centenar de publicaciones en revistas científicas, capítulos de libros, libros y ponencias a congresos. Coordinador del Máster Oficial Interuniversitario en Cultura de Paz, Conflictos, Educación y Derechos Humanos en la Universidad de Málaga. Premio Internacional de Educación Social Joaquím Grau i Fuster sobre la práctica educativa (2012) por la obra titulada "La educación social en territorios periféricos". ORCID ID: https://orcid.org/0000-00032163-8946. Email: victorsolbes@uma.es 